\title{
Dosimetric comparison of 3D conformal conventional radiotherapy versus intensity-modulated radiation therapy both in conventional and high dose radiation for prostate carcinoma
}

\author{
Azza M. Nasr, MD, Rasha A. Elawady, PhD \\ ${ }^{1}$ The Department of Radiation Oncology, National Cancer Institute, Cairo University, Cairo, Egypt., \\ ${ }^{2}$ Department of Radiotherapy \& Nuclear Medicine, National Cancer Institute, Cairo University, \\ Cairo, Egypt.
}

Background: Radiotherapy plays an important role in the management of prostate cancer. There are ongoing efforts to deliver the optimum radiation dose to the prostate while minimizing it to at risk pelvic structures.

Aim: To compare the dose coverage of planning target volume (PTV) and organs-at-risk (OAR) between intensity-modulated radiation therapy (IMRT) and conventional three dimensional conformal radiotherapy (3D-CRT) for the same plan of prostate cancer patients in both conventional dose and high dose.

Methods: Plans of 15 prostate cancer patients with low- or intermediate risk, treated by 3D-CRT, were compared with IMRT to the same dose and to a higher dose. We used XIO- treatment planning system for 3D-CRT and Monaco treatment planning system for IMRT.

Results: Mean and minimum dose to PTV were equal, but the mean maximum dose was significantly higher in IMRT (72Gy) than 3D-CRT. Doses to critical organs in IMRT plans were lower than 3D-CRT, even in the increased dose of $80 \mathrm{~Gy}$.

Conclusion: IMRT is recommended as the standard of care in treatment of prostate carcinoma. Applying escalated dose is recommended.

Key words: Prostate cancer, IMRT, escalated dose.

Corresponding author: Azza Nasr, National Cancer Institute, Cairo University,

E-mail: nasrazza2@hotmail.com, Tel: +20 1121893160

Received: 7-Oct-2015, Accepted: 8-Oct-2015

\section{INTRODUCTION}

Radiotherapy alone or combined with surgery and /or hormonal therapy, plays an important role in the treatment of different risk groups of prostate cancer ${ }^{1}$. It is now generally accepted by most clinicians, in the treatment of prostate cancer ${ }^{2}$. This had originated primarily from retrospective and prospective nonrandomised studies. This has been translated into improved biochemical control by means of permitting dose escalation ${ }^{3,4}$.

Increasing the radiation dose to the prostate was associated with better disease- free survival in the M.D. Anderson and Medical Research Council trials ${ }^{5,6}$. While the results of these trials were encouraging, they were not designed to identify overall survival differences.

The impact of radiation dose escalation on overall survival of prostate cancer patients will be determined by studies in which overall survival was set as a primary endpoint like the Radiation Therapy Oncology Group (RTOG) 0126 trial.
The value of dose escalation, however, is limited by the radiation dose delivered to the organs at risk (OAR); the bladder and the rectum. This emphasizes the importance of minimizing the margins added to the clinical target volume (CTV) when accounting for patient position and internal organ motion variations ${ }^{7}$.

In the past two decades several technological innovations have developed the planning and delivery of radiotherapy, thereby allowing for dose escalation to the prostate. The recent current "standard" dose of primary radiotherapy for prostate cancer is now between $75 \mathrm{~Gy}$ and $80 \mathrm{~Gy}$, which is $10 \%$ to $20 \%$ higher than the safely delivered by conventional radiation ${ }^{8,9}$. Sequential dose escalation studies conducted at Memorial Sloan Kettering Cancer Centre support the notion that the use of intensity-modulated radiation therapy (IMRT) can reduce morbidity compared with three-dimensional conformal radiotherapy (3D-CRT) ${ }^{10}$. A report of the longterm toxicity outcomes with IMRT corroborated a lower 
morbidity profile after high dose IMRT, with an observed incidence of grade 2 rectal and urinary toxicities at $2 \%$ and $15 \%$, respectively ${ }^{11}$.

The same low toxicity profile was obtained in preliminary toxicity analysis of the RTOG 0126 Prostate Cancer Trial ${ }^{12}$.

Our centre routine is to use 3D-CRT for treatment of prostate cancer patients for a conventional dose of 72 Gy. In this study, we aimed to compare the 3D-CRT with IMRT in terms of critical organ sparing and dose homogeneity in conventional dose (IMRT 72 Gy) and in higher dose (IMRT 80Gy).

\section{METHODS}

This dosemetric study included 15 patients with pathologically proven low and intermediate risk prostate cancer. They were treated with 3D-CRT, using the conventional dose of $72 \mathrm{~Gy}$, at Radiation Oncology Department of the National Cancer Institute, Cairo University between January 2015 and June 2015. For each patient, two additional plans were designed, both are IMRT. The first used the conventional dose (72Gy), while the second with high dose (80Gy).

The three plans: 3D-CRT (the one the patient was treated by) and the two IMRT plans were compared regarding critical organ doses and target volume dose homogenization.

Before simulation, patients were asked to drink water and as soon as they had urgency, computed tomography (CT) images with $2.5 \mathrm{~mm}$ slice thickness were obtained in the planning unit. This was followed by magnetic resonance imaging (MRI) scan of the prostate in the radiology department. The axial T2 images were used to determine the prostate volume. Contouring was automatic for the body and manual for the adjacent organs at risk. The CTV included the prostate plus the $1^{\text {st }} 9 \mathrm{~mm}$ of the seminal vesicles. The entire seminal vesicle was included in the CTV if they were involved pathologically. An $8 \mathrm{~mm}$ expansion of the CTV in all directions represented the planning target volume (PTV), except for the posterior margin which extended for only $5 \mathrm{~mm}$. The limits of PTV planning ranged between 95 and 105\%. The critical tolerance dose for the rectum was V70 (the volume receiving 70 Gy) in $\leq 20 \%$ of all volume, V60 in $<35 \%$ and V45 in $<50 \%$. For the bladder: V70 in $\leq 25 \%$ of all volume and $\mathrm{V} 60$ in $<35 \%$. For the femoral heads, the V50 in $\leq 5 \%$ of all volume and for the penile bulb the mean dose should be $<50$ Gy.

An axial IMRT planning slice of a patient is shown in Figure 1.

Statistical analyses were performed in SPSS version 22.0 software (Chicago, ILL, USA) and SAS version 9.4. Significance level was set at $P<0.05$.

\section{RESULTS}

The study included plans of 15 patients of low and intermediate risk. We compared PTV; minimum, maximum and mean doses of IMRT (72Gy) plan and 3D-CRT plan. Although the maximum dose in IMRT (72) was statistically different from that of 3D-CRT, yet there was no difference in the mean dose (Table 1).

Major critical structures that needed to be spared in prostate cancer radiotherapy are rectum, bladder, femoral heads and penile bulb. Although lower in IMRT (72) mean rectum V45, V60 and V70 percentage values were compared, and no statistically significant differences were found between both techniques of the same dose. On escalating the dose to $80 \mathrm{~Gy}$, rectal doses remained similar to that received by lower total dose of 72 Gy (Table 2).

Mean bladder V60 percentage DVH values was less in IMRT (72), however, not statistically significant (Table 3).

Penile bulb was secured markedly, reaching statistically significant difference, with a P- value of 0.009 (Table 4).

Regarding the femur, the volume that 50Gy reached was $0 \%$ in all cases. Mean dose was significantly higher in 3D-CRT (Table 5). 


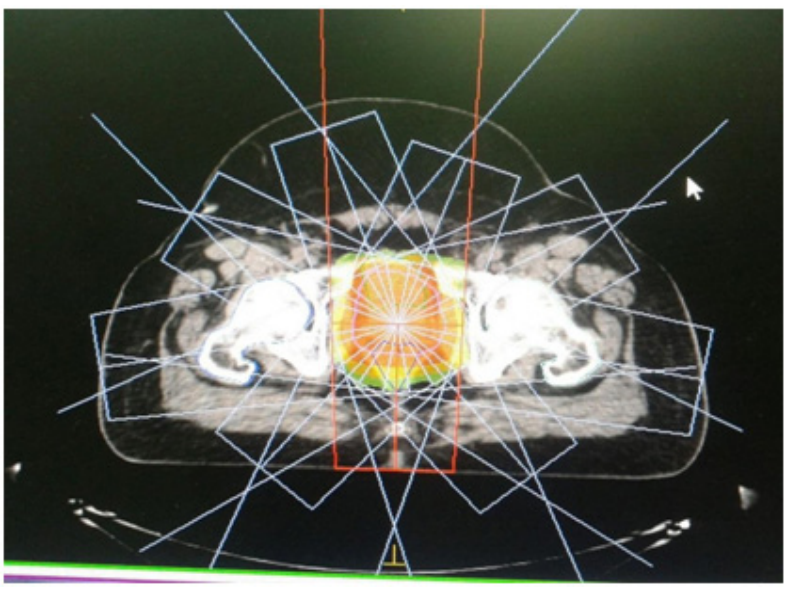

Figure 1: Axial IMRT planning slice of a patient in the study

Table 1: Mean PTV minimum and maximum values of IMRT and 3D-CRT

\begin{tabular}{lcccc}
\hline & 3D-CRT(72) & IMRT(72) & P- value & IMRT(80) \\
\hline Min & 59.5 (SD 3.4) & $59.5($ SD 3) & 0.980 & 66 (SD 4.6) \\
\hline Max & 74.8 (SD 1.6) & 76 (SD1.87) & 0.023 & 85.5 (SD 1.99) \\
\hline Mean & $70.9($ SD 1.04) & $70.6($ SD 0.79) & 0.349 & 78 (SD 0.69) \\
\hline
\end{tabular}

Table 2: Mean \% V45, V60 and V70 values of rectum in IMRT and 3D-CRT.

3D-CRT(72), \% $\quad$ IMRT(72), \% P-value IMRT(80), \%

\begin{tabular}{lcccc}
\hline V70 & $3.9($ SD 3.5) & $3.6($ SD 3.7) & 0.67 & 9.5 (SD 4.5) \\
\hline V60 & $14($ SD 9.8) & 12.4 (SD 4.8) & 0.435 & 16.7 (SD 5.8) \\
\hline V45 & 31 (SD 12) & 28 (SD 5) & 0.405 & 31 (SD 5.6) \\
\hline
\end{tabular}

Table 3: Mean \% V60 and V70 values of bladder in IMRT and 3D-CRT.

\begin{tabular}{ccccc}
\hline & 3D-CRT(72), \% & IMRT(72), \% & P-value & IMRT(80), \% \\
\hline V70 & $13.1($ SD 13.4) & $9.3($ SD 6.1) & 0.334 & 23.6 (SD 18.3) \\
\hline V60 & 28.6 (SD 22.3) & 27.6 (SD 18.9) & 0.548 & 30.9 (SD 18.5) \\
\hline
\end{tabular}

Table 4: Mean dose to penile bulb.

\begin{tabular}{lllll}
\hline & 3D-CRT(72) & IMRT(72) & P-value & IMRT(80) \\
\hline Mean dose & 54 (SD 12.4) & 43 (SD 12.5) & 0.009 & 45 (SD 13.6) \\
\hline
\end{tabular}

Table 5: Mean dose to both femori

\begin{tabular}{lcccc}
\hline & 3D-CRT(72) & IMRT(72) & P-value & IMRT(80) \\
\hline $\begin{array}{l}\text { Right } \\
\text { femur }\end{array}$ & 40.9 (SD 2.923) & 18.7 (SD .311) & 0.0001 & 19.98 (SD 2.001) \\
\hline $\begin{array}{l}\text { Left } \\
\text { femur }\end{array}$ & $42.6(\mathrm{SD} 3.098)$ & $19.09(\mathrm{SD} 4.037)$ & 0.0001 & 20 (SD 2.309) \\
\hline
\end{tabular}

\section{DISCUSSION}

IMRT has long been standard of care in the treatment of patients with prostate cancer as a viable alternative to surgery and its implementation is emerging ${ }^{13}$.

Many comparative studies of IMRT and 3D-CRT showed that IMRT was therapeutically superior to 3D-CRT in prostate cancer treatment ${ }^{11,14}$. In this current work, the plans of 15 patients treated with 3D-CRT were compared with plans created to the same dose using IMRT. The maximum dose to PTV in IMRT of 72Gy plans was significantly higher than that of 3D-CRT with a $P$ - value of 0.02 , yet there was no difference in the mean dose. Although all the figures of the volumes of the bladder and rectum receiving $70 \mathrm{~Gy}$ and $60 \mathrm{~Gy}$ and volume of rectum receiving 45Gy were lower in IMRT $72 \mathrm{~Gy}$ arm than their corresponding in 3D-CRT yet P-value was not significant. This may be attributed to low number of patients included. The doses to both femora and that to penile bulb in favour of IMRT revealed high statistically significant difference, whereas the $\mathrm{P}$ values were 0.001 and 0.009 , respectively. In a similar study, Uysal et al. ${ }^{15}$ compared between IMRT and 3D-CRT dosimetric plans for a 68 Gy dose in 20 patients with low/moderate risk prostate cancer. They found a statistically significant better target organ dose homogeneity with the 7-field IMRT plan $(<0.05)$. The average V40 and V60 rectal dose volume histograms (DVH) differed significantly in favour of IMRT $(p<0.05)$ but not the V25 $(p>0.05)$. Only mean bladder V60 percentage DVH values comparing IMRT and 3D-CRT arms were statistically significant $(p<0.05)$, while comparative mean V25 and V40 values were statistically insignificant $(p>0.05)$. Both mean right and left femoral head dose values were significantly lower in IMRT arm $(p<0.05)$. Similar results have been revealed in previous studies ${ }^{13,16,17}$.

Several single institution series have reported a reduction in late toxicity with the introduction of IMRT compared to 3D-CRT, even with dose escalation ${ }^{11,18,19}$. As higher radiation doses for patients with clinically localized prostate cancer are now considered standard of care ${ }^{20}$, so we conducted third plan using IMRT but for 
Research in Oncology

Vol. 12 | No. $1 \quad 2016$

IMRT vs. 3D-CRT in prostate cancer

escalated dose. In spite of increasing the dose to 80Gy, the dose to critical organs were still nearly the same as that with the IMRT72 . The only exception was that the V70 rectal and bladder doses are higher, but they were still within the constrains for the rectum $(<20 \%)$ and the bladder $(<25 \%)$.

The RTOG 0126 trial compares conventional dose (70.2 Gy) radiation to high dose (79.2 Gy) one in the management of intermediate risk prostate cancer. In the high dose arm of the study, the volumes of the bladder and rectum receiving 65, 70 and 75 Gy were significantly smaller among patients treated with IMRT when compared to those treated with 3D-CRT (all $p<0.0001)^{21}$. The incidence of acute gastrointestinal and genitourinary toxicities was significantly lower with $\mathrm{IMRT}^{12}$.

In another study, prostate cancer patients who were treated with IMRT to high doses (78- 82 Gy) had significantly lower gastrointestinal toxicity in comparison to patients treated with 3D-CRT to lower doses (70- 74 Gy). The volume of the rectum receiving low/intermediate radiation doses was significantly lower in the IMRT group ${ }^{22}$.

The larger the volumes of the rectum receiving high radiation dose, the more frequent the late toxicities. In the MD Anderson trial, the rate of $\geq$ grade 2 complications increased from $16 \%$ to $46 \%$ when $\geq 25 \%$ of the rectum received $>70 \mathrm{~Gy}^{23}$. Our study showed that the volume of the rectum received 70 Gy was $9.5 \pm 4.5 \%$ in IMRT 80 plans.

\section{CONCLUSION}

Increasing the dose hasn't been associated with increase of the dose to organs at risk on using IMRT. When planning radiation therapy, keeping the volume of rectum exceeding $70 \mathrm{~Gy}$ to less than $15 \%-20 \%$. Because higher radiation doses for patients with clinically localized prostate cancer are now considered standard of care, we recommend start using IMRT with high dose .Clinical evaluation of toxicities with high dose regimens should be investigated.

\section{REFERENCES}

1. Hanks GE, Hanlon AL, Epstein B, et al. Dose response in prostate cancer with 8-12 years' follow-up. Int J Radiat Oncol Biol Phys. 2002; 54:427- 435.

2. Trada Y, Plank A and Martin J. Defining a dose-response relationship for prostate external beam radiotherapy. J Med Imaging Radiat Oncol. 2013; 57:213-246.

3. Vicini F, Kestin L, Ghilezan M et al. Radiation dose for prostate cancer: Is more better? Nat Clin Pract Oncol. 2006; 3:298-299.

4. Park SS, Yan D, McGrath S, et al. Adaptive image-guided radiotherapy (IGRT) eliminates the risk of biochemical failure caused by the bias of rectal distension in prostate cancer treatment planning: clinical evidence. Int J Radiat Oncol Biol Phys. 2012; 83: 947-952.

5. Dearnaley DP, Sydes MR, Graham JD, et al. Escalated-dose versus standard- dose conformal radiotherapy in prostate cancer: first results from the MRC RT01 randomised controlled trial. Lancet Oncol. 2007; 8:475-487.

6. Kuban DA, Tucker SL, Dong L, et al. Long-term results of the M. D.Anderson randomized dose-escalation trial for prostate cancer. Int J Radiat Oncol Biol Phys. 2008; 70:67-74.

7. Heemsbergen WD, Hoogeman MS, Witte MG, et al. Increased risk of biochemical and clinical failure for prostate patients with a large rectum at radiotherapy planning: Results from the Dutch trial of $68 \mathrm{GY}$ versus 78 Gy. Int J Radiat Oncol Biol Phys. 2007; 67:1418-1424.

8. Al-Mamgani A, van Putten WL, Heemsbergen WD, et al. Update of Dutch multicenter dose escalation trial of radiotherapy for localized prostate cancer. Int J Radiat Oncol Biol Phys. 2008; 72:980-988.

9. Dearnaley DP, Jovic G, Syndikus I, et al. Escalated-dose versus control-dose conformal radiotherapy for prostate cancer: long-term results from the MRC RT01 randomised controlled trial. Lancet Oncol. 2014; 15:464-473.

10. Zelefsky MJ, Fuks Z, Hunt M, et al. High dose intensity modulated radiation therapy for prostate cancer: early toxicity and biochemical outcome in 772 patients, Int $\mathrm{J}$ Radiat Oncol Biol Phys. 2002; 53:1111-1116.

11. Zelefsky MJ, Chan H, Hunt M, et al. Long-term outcome of high dose intensity modulated radiation therapy for patients with clinically localized prostate cancer. J Urol. 2006; 176:1415-1419.

12. Michalski JM, Yan Y, Watkins-Bruner D, et al. Preliminary toxicity analysis of 3-dimensional conformal radiation therapy versus intensity modulated radiation therapy on the high-dose arm of the Radiation Therapy Oncology Group 0126 prostate cancer trial. Int J Radiat Oncol Biol Phys. 2013; 87:932-938.

13. Zelefsky M, Fuksa Z, Happersett L, et al. Clinical experience with intensity modulated radiation therapy (IMRT) in prostate cancer. Radiother Oncol. 2000; 55:241-249.

14. Latorzeff I, Mazurier J, Boutry C, et al. Benefit of intensity modulated and image-guided radiotherapy in prostate cancer. Cancer Radiother. 2010; 14:479-487.

15. Uysal B, Beyzadeoğlu M, Sager O, et al. Dosimetric evaluation of intensity modulated radiotherapy and 4-field 3 -d conformal radiotherapy in prostate cancer treatment. Balkan Med J. 2013; 30:54-57.

16. Lee CT, Dong L, Ahamad AW, et al. Comparison of treatment volumes and techniques in prostate cancer radiation therapy. Am J Clin Oncol. 2005; 28:618-625

17. Zhu S, Mizowaki T, Nagata Y, et al. Comparison of three radiotherapy treatment planning protocols of definitive external-beam radiation for localized prostate cancer. Int J Clin Oncol. 2005; 10:398-404

18. Jani $\mathrm{AB}, \mathrm{Su} \mathrm{A}$, Correa $\mathrm{D}$, et al. Comparison of late gastrointestinal and genitourinary toxicity of prostate cancer patients undergoing intensity- modulated versus conventional radiotherapy using localized fields. Prostate Cancer Prostatic Dis. 2007;10:82-86

19. Shu HK, Lee TT, Vigneauly E, et al. Toxicity following high-dose three- dimensional conformal and intensitymodulated radiation therapy for clinically localized prostate cancer. Urology. 2001; 57:102-107.

20. Fonteyne V, Ost P, Vanpachtenbeke F, et al. Rectal toxicity after intensity modulated radiotherapy for prostate cancer: 
Which rectal dose volume constraints should we use? Radiother Oncol. 2014; 113:398-403.

21. Bruner DW, Hunt D, Michalski JM, et al. Preliminary patient-reported outcomes analysis of 3-dimensional radiation therapy versus intensity- modulated radiation therapy on the high-dose arm of the Radiation Therapy Oncology Group (RTOG) 0126 prostate cancer trial. Cancer. 2015; 121:2422-2430.
22. Vanasek J, Odrazka K, Dolezel M, et al. Statistical analysis of dose-volume profiles and its implication for radiation therapy planning in prostate carcinoma. Int J Radiat Oncol Biol Phys. 2013; 86:769-776.

23. Pollack A, Zagars GK, Starkschall G, et al. Prostate cancer radiation dose response: results of the M. D. Anderson phase III randomized trial. Int J Radiat Oncol Biol Phys. 2002; 53:1097-1105. 